\title{
Evaluación de la peligrosidad del tsunami de Chile del 16 de setiembre del 2015 para Costa Rica
}

\section{Hazard assessment of the Chilean tsunami of September 16th, 2015 for Costa Rica}

\author{
Silvia Chacón Barrantes ${ }^{1 *}$
}

\begin{abstract}
RESUMEN
El 16 de setiembre del 2015 un sismo Mw 8.3 en Chile causó un tsunami que afectó únicamente a dicho país. Sin embargo, este sismo motivó la realización de un análisis de peligrosidad de tsunami por parte del Sistema Nacional de Monitoreo de Tsunamis (SINAMOT) para recomendar a la Comisión Nacional de Emergencias (CNE) las medidas a tomar al respecto para Costa Rica. Este evento representó un ejercicio de los procedimientos operativos estandarizados con los que cuenta el SINAMOT y la utilización por primera vez de herramientas locales de pronóstico de alturas y corrientes de tsunamis, además con resultados exitosos. En este artículo se detallan el desarrollo del evento y las herramientas usadas para el análisis de peligrosidad por tsunami, así como los registros que se dieron del tsunami en territorio nacional. Dicho tsunami fue registrado en Costa Rica continental únicamente en mareógrafos, tal y como había sido pronosticado por el SINAMOT. Este artículo tiene un enfoque operativo y muestra los resultados en la misma forma en que se usan durante el evento para la toma de decisiones.
\end{abstract}

Palabras claves: Tsunamis, Tsunami Chile 2015, Costa Rica, Pronóstico de Tsunamis, Alerta de Tsunami.

\begin{abstract}
A Mw 8.3 earthquake took place in Chile on September 16th, 2015 causing a local tsunami. As a consequence, this earthquake prompted the National Tsunami Monitoring System (SINAMOT) in Costa Rica to conduct a tsunami hazard assessment in order to provide the National Emergency Committee (CNE) with recommendations on how to respond locally to the tsunami. This event represented an exercise to implement SINAMOT Standard Operating Procedures (SOPs) and to use local forecast tools for tsunami heights and currents for the first time, which produced successful results. This paper describes the forecast conducted by SINAMOT, the tools used for the hazard assessment, and the tsunami records in Costa Rica. Along Costa Rica's continental shores, the tsunami was recorded only by tide gauge instruments, as predicted by SINAMOT. This paper has an operative approach and shows the results in the same format as they are used for decision making processes during the event.
\end{abstract}

Keywords: Tsunamis, 2015 Chilean Tsunami, Costa Rica, Tsunami Forecast, Tsunami Warning.

\section{INTRODUCCIÓN}

Los tsunamis son ondas superficiales gravitatorias provocadas por una perturbación súbita de la superficie del agua. Más del $80 \%$ de los tsunamis han sido causados por sismos (NGDC/WDS, 2015), aunque pueden ser ocasionados por deslizamientos de tierra, erupciones volcánicas, caídas de meteoritos y ciertas

\footnotetext{
1 Sistema Nacional de Monitoreo de Tsunamis (SINAMOT), Programa Red de Observación del Nivel del Mar e Investigación de Amenazas Costeras (RONMAC), Departamento de Física, Universidad Nacional. silviachaconb@gmail.com*
}

Recibido: 26 de octubre de 2015

Corregido: 19 de enero de 2016

Aceptado: 21 de enero de 2016

DOI: http://dx.doi.org/10.15359/revmar.8-1.8 
perturbaciones atmosféricas. Más del $70 \%$ de los tsunamis mortíferos han ocurrido en el Océano Pacífico (NGDC/ WDS, 2015), aunque se han registrado tsunamis en todos los océanos del mundo y en algunos mares y lagos.

En 1960 un sismo con magnitud de momento $\mathrm{Mw} 9.5$ en Chile, el más grande registrado en la historia, causó un tsunami que cruzó el Océano Pacífico sin ningún tipo de advertencia, ocasionando muertes y considerables daños en Hawái y Japón. En 1964 un tsunami originado por un sismo Mw 9.3 en Alaska afectó también otros países en la cuenca del Pacífico. Estos dos tsunamis motivaron la creación en 1965 del Grupo Intergubernamental para la Coordinación del Sistema de Alerta de Tsunamis del Pacífico (ICG/PTWS, por sus siglas en inglés), perteneciente a la Comisión Oceanográfica Internacional de la UNESCO (UNESCO, 2015). Este sistema de coordinación entre países tiene un centro operativo, el Centro de Alerta de Tsunamis del Pacífico(PTWC) ubicado en Hawái, que monitorea la ocurrencia de sismos costeros que podrían ocasionar tsunamis y facilita la información a los países miembros, quienes deben analizarla y tomar sus propias decisiones.

Costa Rica pertenece al ICG/ PTWS, por lo tanto, recibe los boletines de tsunamis del PTWC a través de su Punto Focal Nacional de Tsunamis (TWFP, por sus siglas en inglés), que es la Comisión Nacional de Prevención de Riesgos y Atención de Emergencias (CNE). Costa Rica también pertenece al ICG/CARIBE-
EWS, el Grupo Intergubernamental para la Coordinación del Sistema de Alerta de Tsunamis del Caribe, cuyo centro operativo es también el PTWC.

En Costa Rica se han registrado 29 tsunamis, 24 de ellos en la costa pacífica y cinco en la costa caribeña. De los 24 tsunamis del Pacífico, 11 de ellos han sido de origen local y 13 de origen remoto. La gran mayoría de registros de tsunamis remotos son únicamente instrumentales, con excepción del tsunami de Colombia de 1906 que causó una inundación visible en Playa Potrero y de los registros en la Isla del Coco $(2007,2010$ y 2011), donde parece haber un efecto de resonancia en la desembocadura del río Genio. Sin embargo, el registro histórico que llevamos de tsunamis en Costa Rica se remonta aproximadamente a 300 años atrás. Este período de tiempo resulta muy corto en escalas geológicas, por lo que no es posible descartar la llegada al país de un tsunami con consecuencias catastróficas. La población en las costas de nuestro país y el desarrollo turístico de estas han experimentado un crecimiento exponencial en las últimas décadas, aumentando la cantidad de personas expuestas ante un eventual tsunami.

Es por esto que en el año 2007, el Programa Red de Observación del Nivel del Mar e Investigación de Amenazas Costeras (RONMAC) del Departamento de Física de la Universidad Nacional inició una colaboración con la CNE para brindarle a esta asesoría en el tema de alertas de tsunamis. Sin embargo, dicha colaboración se daba de manera informal y con protocolos establecidos 
únicamente para la comunicación entre la CNE y el RONMAC, lo que podría tener consecuencias negativas en el caso de que un tsunami lejano afectara a nuestras costas. Debido a esto, en abril del 2014 se creó el Sistema Nacional de Monitoreo de Tsunamis (SINAMOT) como una actividad académica conjunta entre el Programa RONMAC-UNA y la Unidad de Mares, Ríos y Estuarios (IMARES) del Instituto de Investigaciones en Ingeniería (INII) de la Universidad de Costa Rica (UCR).

Cada vez que la CNE como TWFP recibe una comunicación del PTWC con respecto a la ocurrencia de un sismo costero que podría generar tsunami, se comunica con el SINAMOT, quien realiza en forma inmediata un análisis de la peligrosidad del eventual tsunami para nuestras costas y lo comunica a la CNE con las respectivas recomendaciones. El SINAMOT cuenta con procedimientos operativos estandarizados (SOP, por sus siglas en inglés) para el análisis de la peligrosidad por tsunami debido a sismos costeros en el Pacífico y el Caribe, los que se ejecutan cada vez que se recibe un boletín del PTWC $\mathrm{y}$ cada vez que sucede un sismo con Mw mayor a 6.0 en nuestras costas. Aunque para tsunamis locales la afectación es muy localizada y, por lo tanto, los tiempos de arribo son sumamente reducidos, por ejemplo, para la Península de Nicoya se calculan en menos de 10 min (ChacónBarrantes \& Protti, 2011) y el tsunami de Limón de 1991 llegó entre 3 y 5 min después del sismo a Puerto Viejo. El SINAMOT ha sido nombrado por el Gobierno de Costa Rica como Contacto Nacional de Tsunami (TNC, por sus siglas en inglés) ante el ICG/ PTWS y el ICG/CARIBE-EWS, lo que implica que el SINAMOT es la entidad encargada de representar a Costa Rica en la coordinación de actividades internacionales de alerta y mitigación de tsunamis.

Hasta octubre del 2015, el SINAMOT ha atendido 84 boletines de tsunamis emitidos por el PTWC, 75 de ellos para la costa pacífica y nueve para la costa caribeña. Únicamente nueve de estos sismos ocasionaron tsunamis y solo de uno de ellos hay registro en nuestro país.

El 16 de setiembre del 2015 a las 4:55 p.m., hora de Costa Rica, sucedió un sismo $\mathrm{Mw} 8.3$ frente a las costas de la región central de Chile, el cual motivó el envío de un boletín por parte del PTWC a todos los países miembros del ICG/PTWS. En este artículo se detallan los procedimientos que se siguieron para analizar la peligrosidad por tsunami para nuestro país debido a dicho sismo, enfocándose en el uso por primera vez de nuestras propias herramientas de pronóstico de alturas y corrientes de tsunami.

\section{MATERIALES Y MÉTODOS}

\section{Procedimientos de análisis de peligrosidad}

La activación del SINAMOT se dio con una llamada de Base Cero de la CNE a las 5:04 p.m. (hora de Costa Rica) al 
recibir el boletín del PTWC, tal como lo indican los protocolos de comunicación acordados entre ambas instituciones. Los procedimientos operativos estandarizados (SOPs) del SINAMOT para el análisis de peligrosidad por tsunami están basados en:

- Análisis de la información sísmica.

- Análisis de la información histórica de sismos semejantes originados en la misma región.

- Monitoreo del registro del tsunami a su paso por las estaciones del nivel del mar más cercanas al epicentro, siempre y cuando el tiempo de viaje del tsunami lo permita.

El SINAMOT emitió un reporte inicial en el que se indicó que la peligrosidad era nula, ya que en ese momento la magnitud del sismo se había indicado en Mw 7.9 y su profundidad en $33 \mathrm{~km}$. Alrededor de 10 minutos después, el PTWC sube la magnitud del sismo a Mw 8.3 y baja su profundidad a $10 \mathrm{~km}$. Una magnitud mayor del sismo y una profundidad menor implican que había liberado más energía hacia el océano de lo pensado originalmente, incrementando de esa manera el tamaño del tsunami, por lo tanto, se decidió en un segundo reporte incrementar el nivel de peligrosidad a verde hasta no descartar posibles corrientes peligrosas. Con base en experiencias pasadas de tsunamis originados en Chile se sabía que una inundación por tsunami en nuestro país era altamente improbable, pero las corrientes peligrosas no se podían descartar aún. En el pronóstico de alturas de tsunami emitido después por el PTWC se incluye a Costa Rica en el grupo de países con alturas pronosticadas entre $30 \mathrm{~cm}$ y $1 \mathrm{~m}$.

Para realizar un pronóstico más detallado de las alturas y velocidades de corriente que el tsunami tendría en Costa Rica se ejecutaron varios modelos numéricos de tsunamis que se detallan a continuación, los cuales se encuentran en fase experimental y, por lo tanto, todavía no forman parte de los SOPs del SINAMOT.

\section{Modelado numérico de tsunamis}

Los tsunamis suelen modelarse como ondas de aguas someras debido a que las ecuaciones de aguas someras son sencillas de resolver numéricamente y son una representación aceptable de la física de los tsunamis (Levin \& Nosov, 2009). La principal diferencia radica en que las ecuaciones de aguas someras no son dispersivas y los tsunamis pueden presentar dispersión después de haber viajado largas distancias o si sus períodos son muy cortos.

Para el modelado de tsunamis se deben conocer las características de la fuente del tsunami, en el caso de tsunamis causados por sismos se requieren los parámetros de ruptura del sismo. Adicionalmente, se necesita la batimetría de la región por la que el tsunami se propaga, que se suele obtener de datos de batimetría globales como GEBCO (British Oceanographic Data Center, 2011). Si se quiere modelar la inundación por tsunami 
y sus alturas en la costa se requieren también la batimetría y topografía de la región de interés.

El SINAMOT cuenta con las siguientes herramientas de modelado numérico que permiten la realización del pronóstico de alturas y corrientes de tsunamis:

- Tsunami Travel Time (TTT).

- Tsunami Forecast System (Tweb).

- Community Model Interface for Tsunami (ComMIT).

El programa Tsunami Travel Time (TTT SDK v 3.3.2r1) fue desarrollado por el Dr. Paul Wessel (Geoware, 2012) y ha sido comprado por el PTWC y el Centro Internacional de Información de Tsunamis (ITIC) para su libre distribución para efectos de alertas de tsunamis. Este programa permite, a partir del epicentro del sismo, calcular el tiempo aproximado de viaje de un tsunami en toda la cuenca y la hora aproximada de arribo a ciertas localidades. Esta herramienta utiliza una fuente puntual centrada en el epicentro del sismo y la propaga usando una batimetría global de baja resolución para obtener tiempos estimados. Adicionalmente, permite cargar las isócronas que calcula en el programa Tide Tool, desarrollado por el PTWC, que monitorea el avance del tsunami por la cuenca respectiva mostrando en tiempo real las lecturas de las estaciones del nivel del mar pertinentes.

El Tsunami Forecast System (Tweb) es una herramienta de modelado de tsunamis basada en la web, que va tomando las lecturas de las boyas de aguas profundas (DART) por las que va pasando el tsunami y realiza una inversión del tsunami para obtener las características aproximadas de la fuente sísmica. Con esas características de la fuente sísmica efectúa el modelado de propagación del tsunami para toda la cuenca para dar un pronóstico. El Tweb fue desarrollado por el Centro de Investigación en Tsunamis (NCTR) de la Agencia Nacional Atmosférica y Oceanográfica de los Estados Unidos de América (NOAA), quien es el que lleva a cabo el procedimiento descrito. Una vez obtenido el pronóstico se puede ver una imagen de este en el website de Tweb, disponible para usuarios autorizados y, además, se hace disponible en el servidor de la NOAA para descargarlo desde ComMIT.

El ComMIT (Community Model Interface for Tsunami) también fue desarrollado por el NCTR de la NOAA para la realización de mapas de evacuación por tsunami por parte de los países del Océano Índico después del tsunami de Indonesia del 2004. El ComMIT ha sido distribuido también en el Océano Pacífico y en el Mar Caribe y, adicionalmente, permite descargar del servidor de Tweb la fuente sísmica de un tsunami en curso para hacer un modelado de inundación de tsunami para pronóstico en una región previamente configurada y validada por registros de tsunamis anteriores. El ComMIT presenta sus resultados en forma de imágenes y animaciones en su misma interfaz y permite exportar las imágenes en 
formato KMZ para abrirlas en el programa Google Earth. En el caso presente y para efectos de pronóstico, este último formato es el que resulta más útil, ya que permite navegar por los resultados y referenciarlos a las respectivas comunidades costeras en forma sencilla, lo que facilita la toma de decisiones que, por lo general, se debe hacer de manera muy rápida.

En el caso del tsunami de Chile del 2015, el sismo ocurrió a las 4:55 p.m., hora de Costa Rica, y a las 6:51 p.m., hora de Costa Rica, dos horas después, se hizo disponible la fuente sísmica en el servidor de Tweb. Dicha fuente sísmica se usó en ComMIT en un modelo de inundación por tsunami de baja resolución.

Este modelo de baja resolución se está usando en el Proyecto de Investigación SIA-0569-13, adscrito al Programa RONMAC-UNA a solicitud de la CNE, para realizar mapas de evacuación por tsunami. El modelo consta de dos mallas anidadas, la primera con una resolución de 60 arcsec cubriendo toda la costa pacífica de Costa Rica, incluyendo la Isla del Coco, y la segunda con una resolución de 12 arcsec cubriendo el Pacífico Norte y Central de Costa Rica (Fig. 5). Para estas mallas se utilizaron datos de topografía obtenidos mediante LIDAR (INEGI, 2015) en las regiones donde estaban disponibles, en las demás regiones se usaron datos de topografia global obtenidos satelitalmente (Farr et al. 2007). Los datos de batimetría fueron obtenidos de cartas náuticas de diferentes escalas que fueron digitalizadas. Debido a que dichas cartas náuticas son de escalas grandes no fue posible configurar mallas de mayor resolución. El modelo de baja resolución fue validado con registros de los tsunamis históricos de las Islas Salomón del 2013, Japón del 2011, Chile del 2010 y Alaska de 1964 (Chacón-Barrantes \& ArozarenaLlopis, in prep.).

Actualmente, como parte del proyecto de mapas de evacuación se están realizando levantamientos batimétricos en algunas localidades definidas como prioritarias para configurar modelos de alta resolución que además se puedan usar para pronóstico. La validación de estos modelos de alta resolución en las localidades escogidas se hará una vez que se tengan observaciones de tsunamis reales en ellas, mientras tanto, su pronóstico se hará indicando que los modelos no han sido validados.

\section{RESULTADOS Y DISCUSIÓN}

Utilizando el programa Tsunami Travel Time (TTT) se obtuvo que el tsunami tardaría alrededor de seis horas y media en llegar al extremo sur de nuestro país y cerca de 15 minutos menos a la Isla del Coco, según se puede ver en la Fig. 1. Esto quiere decir que el tsunami arribaría a la Isla del Coco aproximadamente a partir de las 11:15 p.m. Se había especificado que la hora aproximada de arribo a los mareógrafos sería a las 11:57 p.m. a Quepos, 12:10 a.m. a Los Sueños y 12:26 a.m. a Papagayo, del mismo miércoles 16 de setiembre.

Por medio del programa Tide Tool se monitorearon las estaciones 
(a)

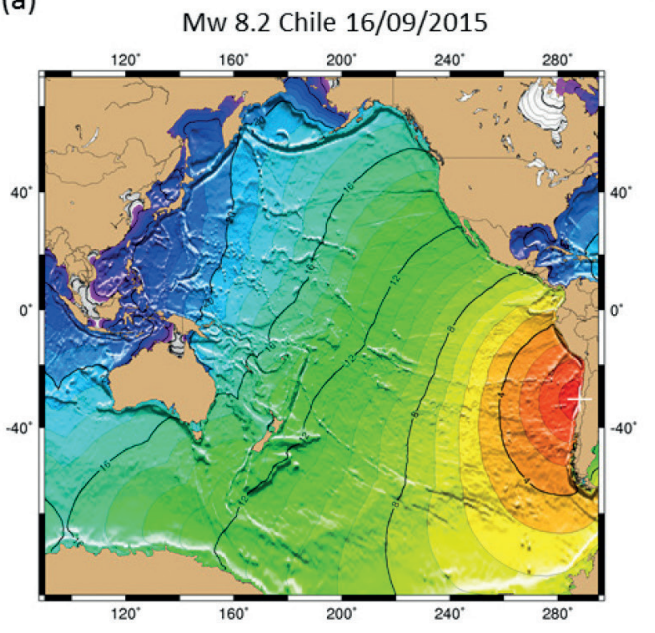

(b)

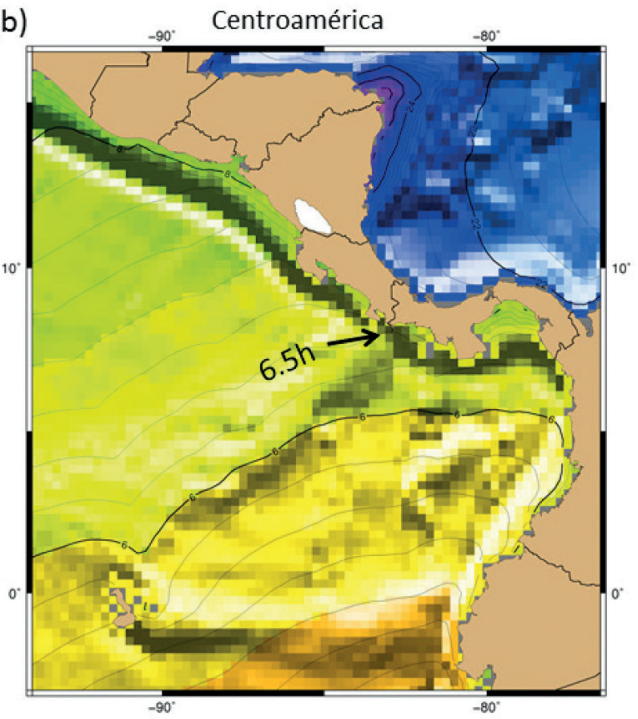

GMT

2015 Sep 16 23:23:40 TSUNAMI TRAVEL TIMES USING POINT SOURCE (EPICENTER)

Fig. 1. Tiempo de viaje del tsunami obtenido con el programa TTT. Para Costa Rica se observa que se tarda entre 6 y 7 horas en llegar, incluyendo a la Isla del Coco Fig. 1. Tsunami travel time obtained using the TTT software. In the case of Costa Rica, the tsunami took between 6 and 7 hours to arrive, including Cocos Island

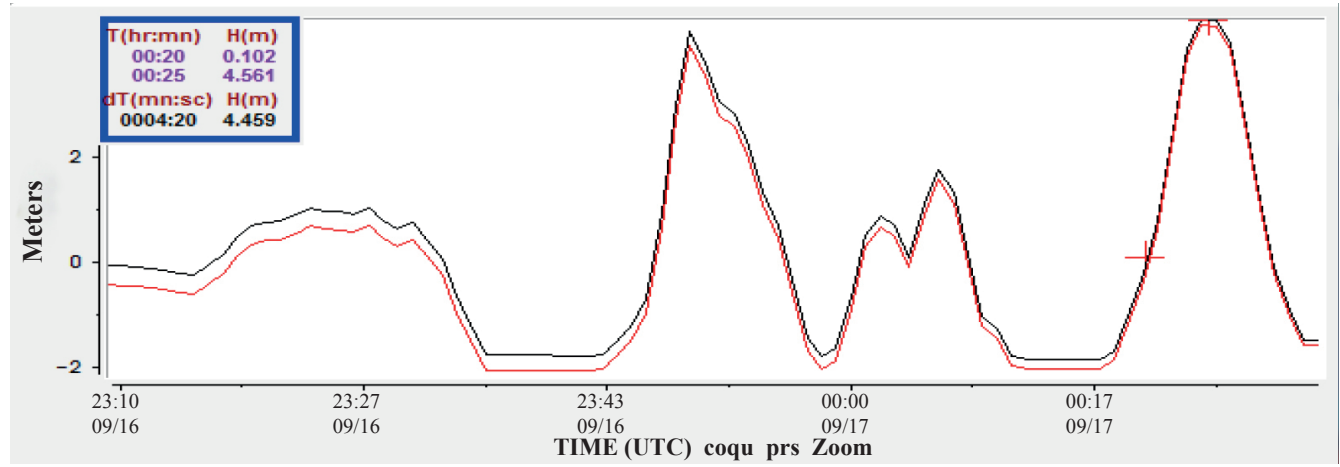

Fig. 2. Registro del tsunami en la estación de Coquimbo, Chile, la más cercana al epicentro del sismo. Se puede observar una altura máxima de tsunami de $4.46 \mathrm{~m}$ desde el nivel medio del mar y una amplitud pico-valle máxima mayor de $6 \mathrm{~m}$. También se puede observar que el mareógrafo se "seca" en dos ocasiones cerca de las 23:43 UTC y las 00:17 UTC, es decir, el tsunami hace retroceder el mar tanto que el instrumento queda fuera del agua varios minutos Fig. 2. Tsunami record from the tide gauge station at Coquimbo, Chile, which is the sea level station closest to the epicenter. The maximum tsunami height observed was $4.46 \mathrm{~m}$ from mean sea level and the maximum peak-to-trough amplitude was over $6 \mathrm{~m}$. The tide gauge "dried out" twice, at approximately 23:43 UTC and 00:17 UTC; that is, the tsunami pushed back the sea to a point that the tide gauge was out of the water for several minutes 


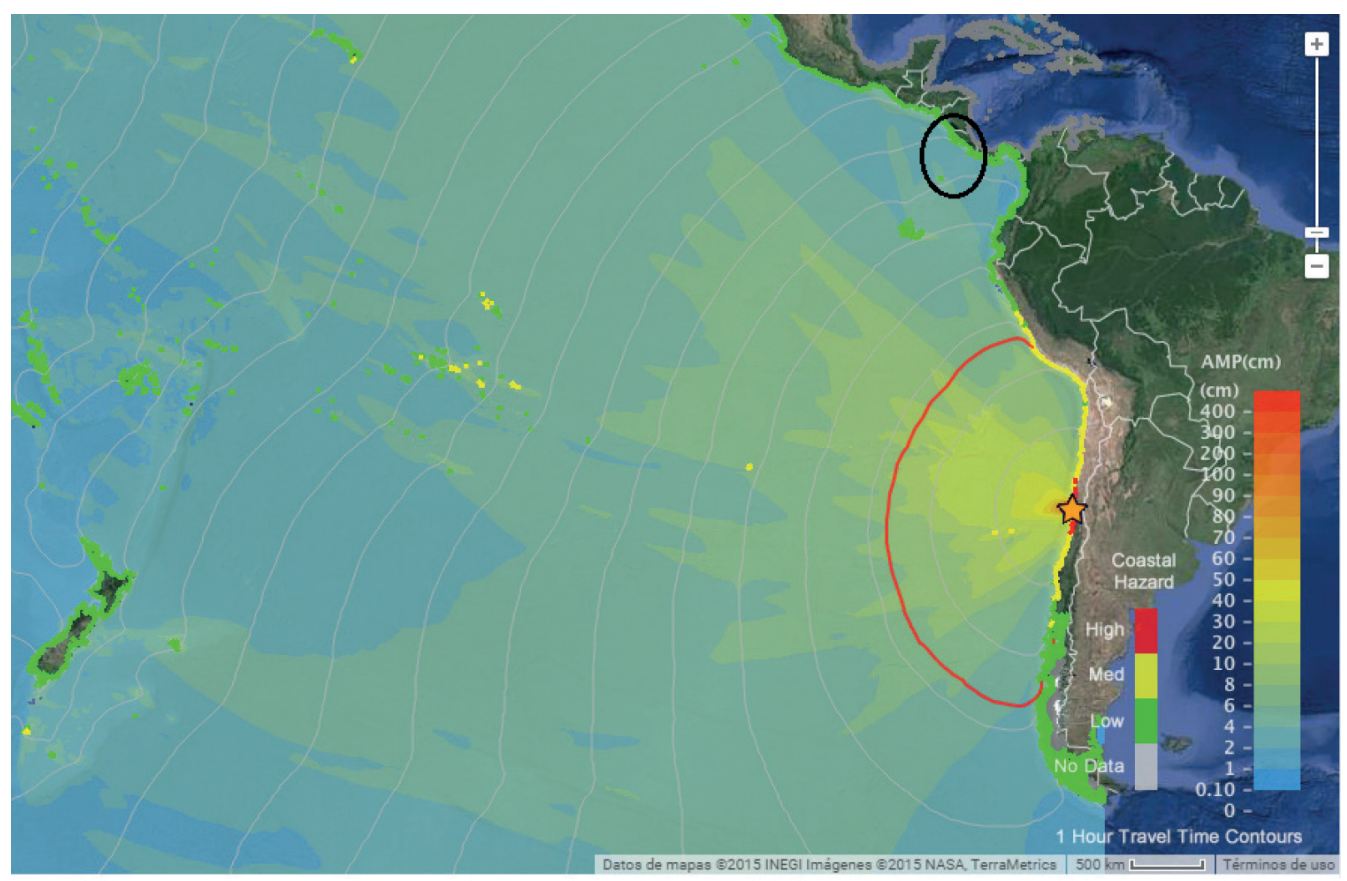

Fig. 3. Imagen tomada de la página del Tweb (NOAA) con las alturas máximas en centímetros del tsunami en aguas profundas. Dichas alturas se obtuvieron del modelado del sismo y tsunami que se hicieron en el NCTR con base en los registros de las boyas DART más cercanas. La línea roja muestra la posición del frente de onda del tsunami en el momento en que la imagen fue capturada. El círculo negro muestra a Costa Rica, incluyendo la Isla del Coco. Se puede ver una rama de energía que se dirige hacia la Isla del Coco

Fig. 3. Screenshot of the Tweb website (NOAA) with tsunami maximum heights in centimeters. Such heights were obtained from the inversion propagation model prepared by NCTR based on records from the closest DART buoys. The red line shows the tsunami front when the image was captured. The black oval shows Costa Rica including Cocos Island. An energy ray is visible pointing towards Cocos Island

del nivel del mar más cercanas al epicentro y alrededor de las 6:30 p.m., hora de Costa Rica, la mayor altura del tsunami había sido registrada en la estación de Coquimbo (Chile) con un valor de $4.46 \mathrm{~m}$ (Fig. 2). Esta altura se considera moderada para haber sido registrada en la estación más cercana al epicentro y, por lo tanto, refuerza el pronóstico de que el tsunami no causaría inundaciones en Costa Rica.
Alrededor de las 7:30 p.m. se descargó la fuente del tsunami del servidor de Tweb y se usó como forzamiento en el modelo de baja resolución en ComMIT para calcular las alturas y corrientes costeras del tsunami y confirmar el pronóstico inicial. En la gráfica del resultado de la propagación del tsunami que hizo Tweb se podía ver una rama de energía que se dirigía directamente hacia la 


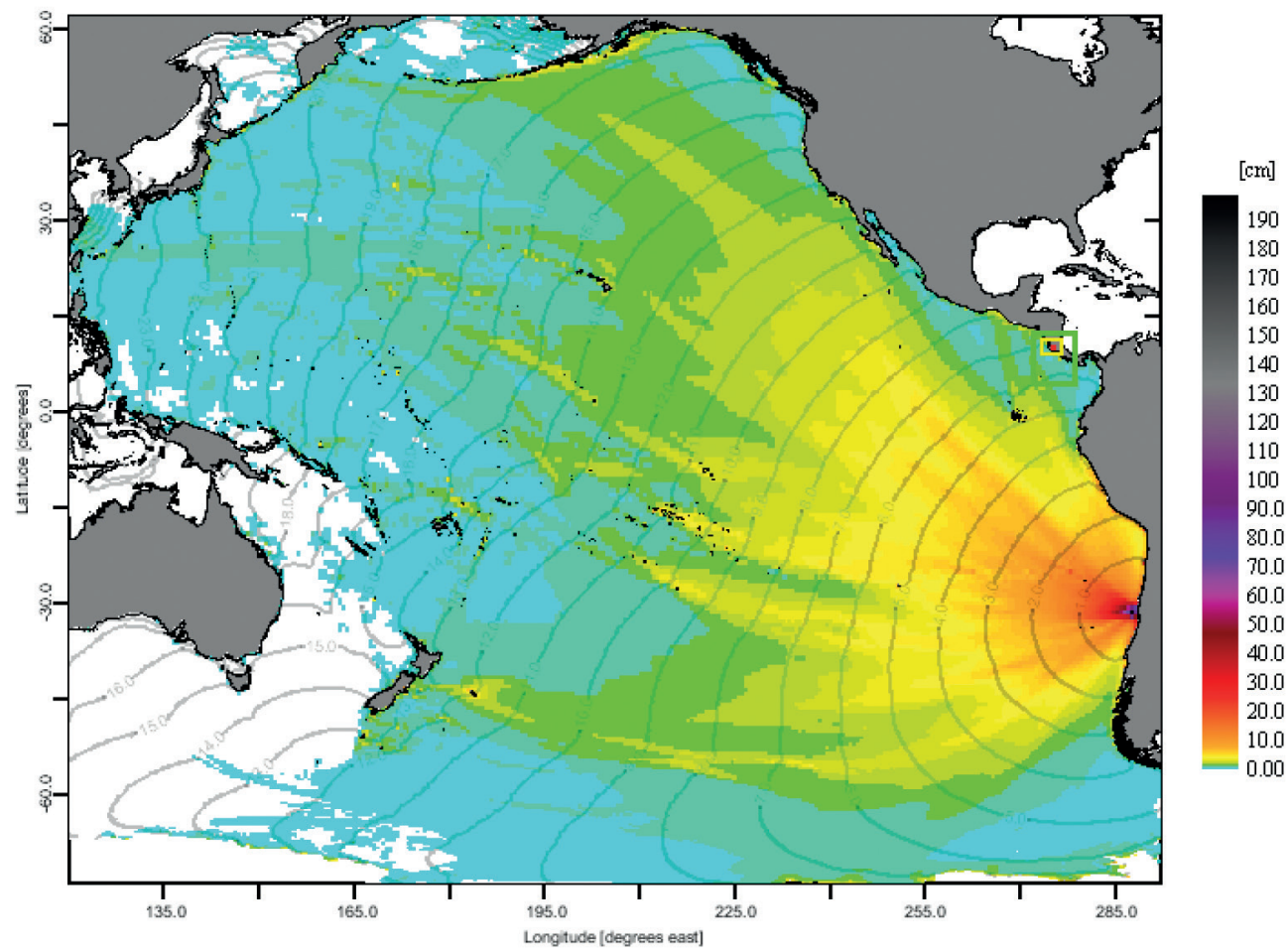

Fig. 4. Alturas máximas en centímetros del tsunami obtenidas por el NCTR (Fig. 3) pero graficadas en el Programa ComMIT. Los contornos son isócronas de la propagación del tsunami en horas

Fig. 4. Maximum tsunami heights in centimeters obtained from NCTR (Fig. 3) plotted with the ComMIT software. The contours represent isochrones of the tsunami propagation in hours

Isla del Coco (Figs. 3, 4 y 5), por lo que se consideró la posibilidad de que ocurrieran corrientes peligrosas en los alrededores de la Isla.

El modelo de baja resolución en ComMIT tardó poco más de una hora en correr y dio como resultado una altura máxima de $56 \mathrm{~cm}$ para toda la costa del Pacífico Norte y Central. En las Figs. 6 y 7 se muestran las alturas máximas que resultaron del modelo de pronóstico de baja resolución en dos localidades, en ambos casos las máximas fueron de alrededor de $20 \mathrm{~cm}$. Dichas figuras se realizaron usando el programa Google Earth, tal como se hizo durante el desarrollo del evento, cuando se requiere visualizar los resultados en forma rápida y sencilla para la toma de decisiones. No se consideraron los pronósticos realizados para Quepos debido a que actualmente el mareógrafo en dicho lugar se encuentra dentro de una marina que es imposible resolver con la escala del modelo actual.

El ComMIT indicó corrientes máximas de cerca de $1.2 \mathrm{~m} / \mathrm{s}$ en las localidades de Puerto Jiménez, Sierpe, 


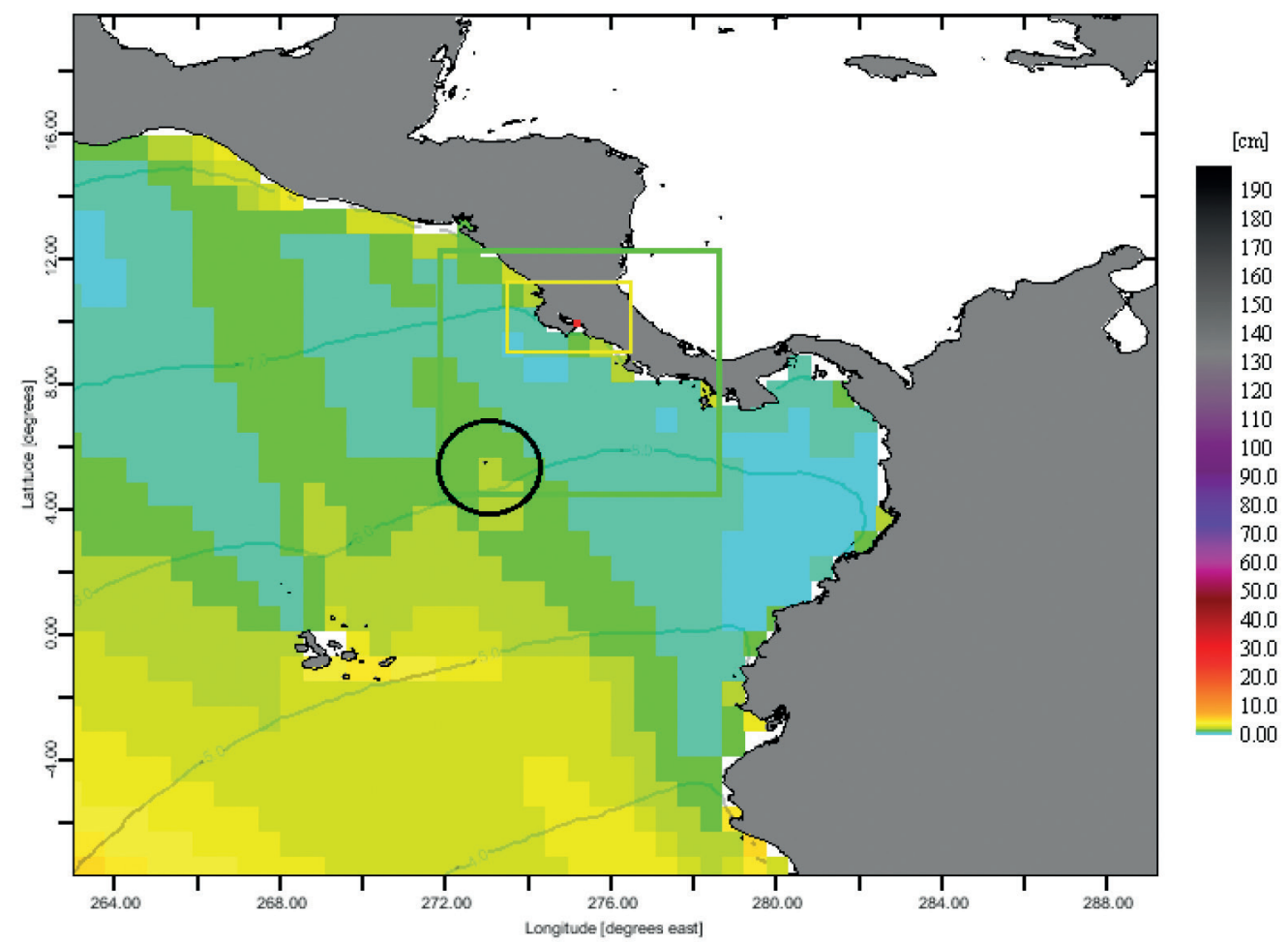

Fig. 5. Acercamiento de la Fig. 4 de las alturas máximas del tsunami en centímetros. En el círculo negro se muestra la Isla del Coco, se puede ver más en detalle la rama de energía del tsunami. Los cuadrados verde y amarillo muestran la extensión de las mallas en las que se modeló con más detalle el tsunami para tener un pronóstico de alturas y corrientes para nuestras costas. El punto rojo muestra la ubicación de la ciudad de Puntarenas. Los contornos son isócronas de la propagación del tsunami en horas

Fig. 5. Close-up of Fig. 4, maximum tsunami heights in centimeters. The black circle shows Cocos Island and a more detailed image of the energy ray. Green and yellow rectangles show the grids where the tsunami was modeled for tsunami heights and currents forecasted for Costa Rica. The red dot shows the location of Puntarenas city. The contours represent isochrones of the tsunami propagation in hours

Parque Marino Ballena, Isla Damas, Bejuco de Pacífico Central, Esterillos, Tárcoles, Bajamar, Bejuco de Pacífico Norte, Camaronal, Sámara y Potrero. Por lo tanto, el SINAMOT recomendó precaución en las localidades mencionadas después de las 11 p.m. Si bien una corriente de alrededor de $1 \mathrm{~m} / \mathrm{s}$ no representa peligro para embarcaciones, sí representa un peligro moderado para bañistas, nadadores y buzos, pero debido a la hora esperada de arribo (cerca de medianoche) era poco probable que se encontrara gente nadando 


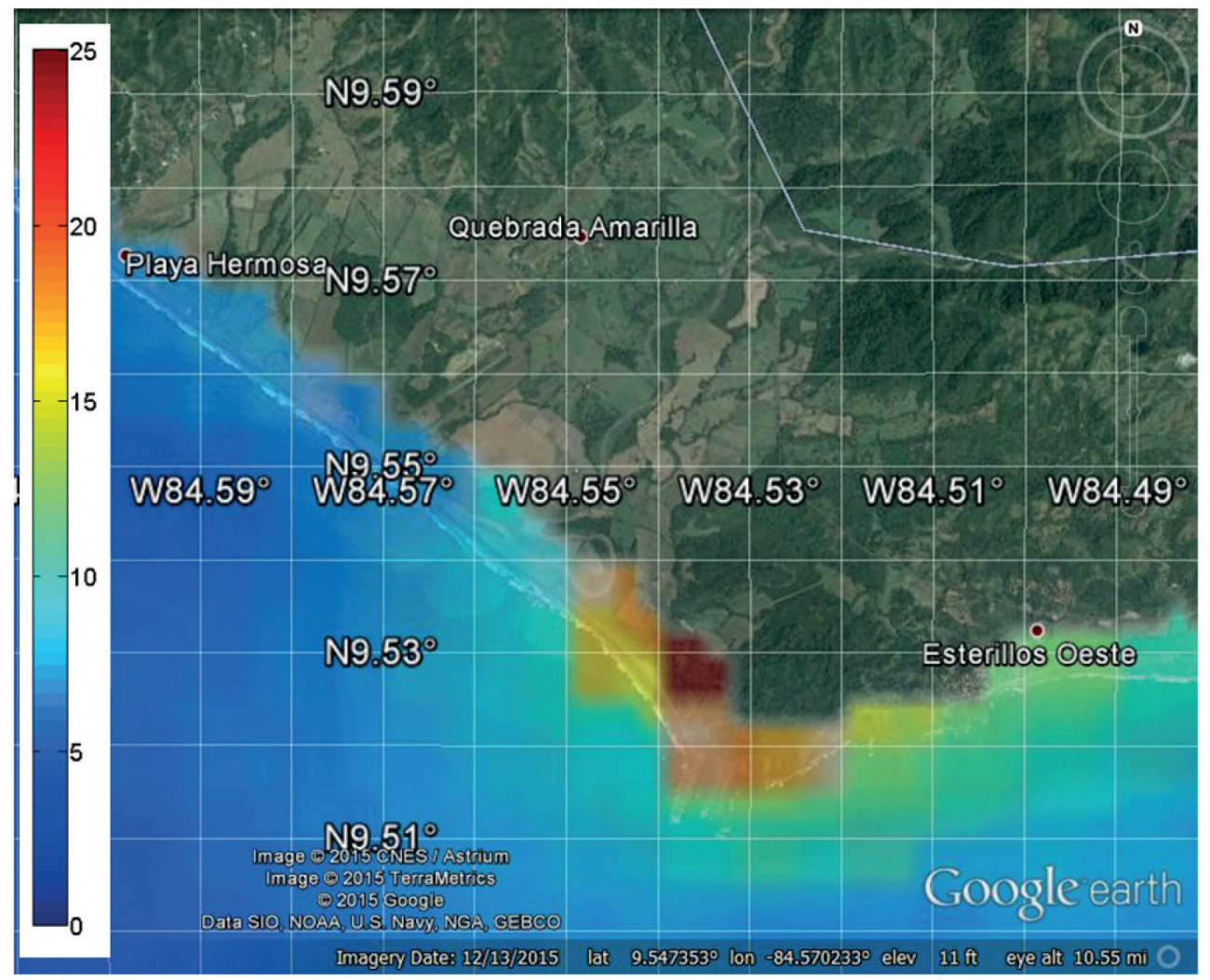

Fig. 6. Acercamiento a las alturas máximas del tsunami obtenidas como resultado del modelo de pronóstico en el este de Playa Hermosa de Jacó y oeste de Esterillos. La escala de colores está en centímetros. El modelo es de baja resolución y por eso hay zonas que parecen estar inundadas aunque en realidad no lo están

Fig. 6. Close-up of maximum tsunami heights resulting from the forecast model at east Hermosa Beach and west Esterillos Beach, in the Central Pacific coast. Color scale is in centimeters. In the figure, given the low resolution of the model, some regions appear to be inundated but they are really not

o buceando en el mar. Para la Isla del Coco, el modelo predijo corrientes por tsunami menores a $10 \mathrm{~cm} / \mathrm{s}$, por lo tanto, el SINAMOT descartó afectación por corrientes para la Isla.

\section{Registros del tsunami en nuestras costas}

El Programa RONMAC-UNA administra los cuatro mareógrafos que existen en Costa Rica (Fig. 8). La llegada del tsunami al mareográfo de Quepos se muestra en las Figs. 9 y 10. Lamentablemente, no se registró el tsunami en las estaciones de Papagayo y Los Sueños debido a fallas técnicas en ambas estaciones. En el mareógrafo de Quepos, el tsunami tuvo una altura máxima de $18 \mathrm{~cm}$ y arribó a las 12:15 a.m. del 17 de setiembre, 18 minutos después de la hora pronosticada. 


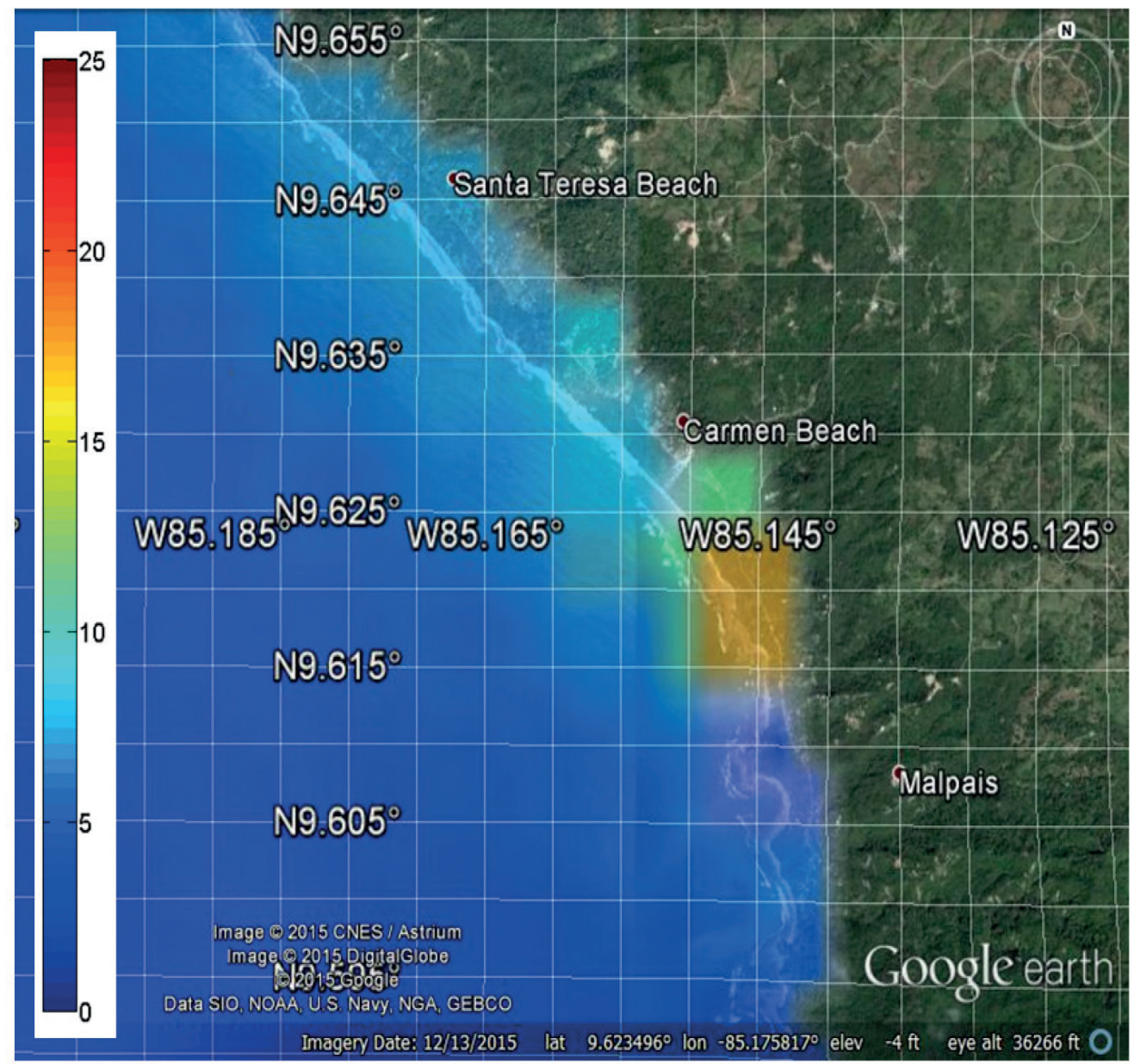

Fig. 7. Acercamiento a las alturas máximas del tsunami obtenidas como resultado del modelo de pronóstico en la zona de Malpaís. La escala de colores está en centímetros. El modelo es de baja resolución y por eso hay zonas que parecen estar inundadas aunque en realidad no lo están Fig. 7. Close-up of maximum tsunami heights obtained from the forecast model at Malpais Beach on south Nicoya Peninsula. Color scale is in centimeters. In the figure, given the low resolution of the model, some regions appear to be inundated but they are really not

Esta diferencia se debe sobre todo a que la calidad y resolución de la batimetría global empleada por el TTT no es la ideal. Adicionalmente, el modelo numérico usado en el TTT no considera la propagación hasta la costa, sino que realiza una aproximación, la que no toma en cuenta bahías, posible corroborarlo para este tsunami.

golfos ni marinas, los cuales por su geomorfología podrían retardar el arribo del tsunami. La autora considera que dicho efecto es probablemente aún mayor para el mareógrafo de Papagayo que se encuentra al final de una bahía de forma alargada, sin embargo, no fue 


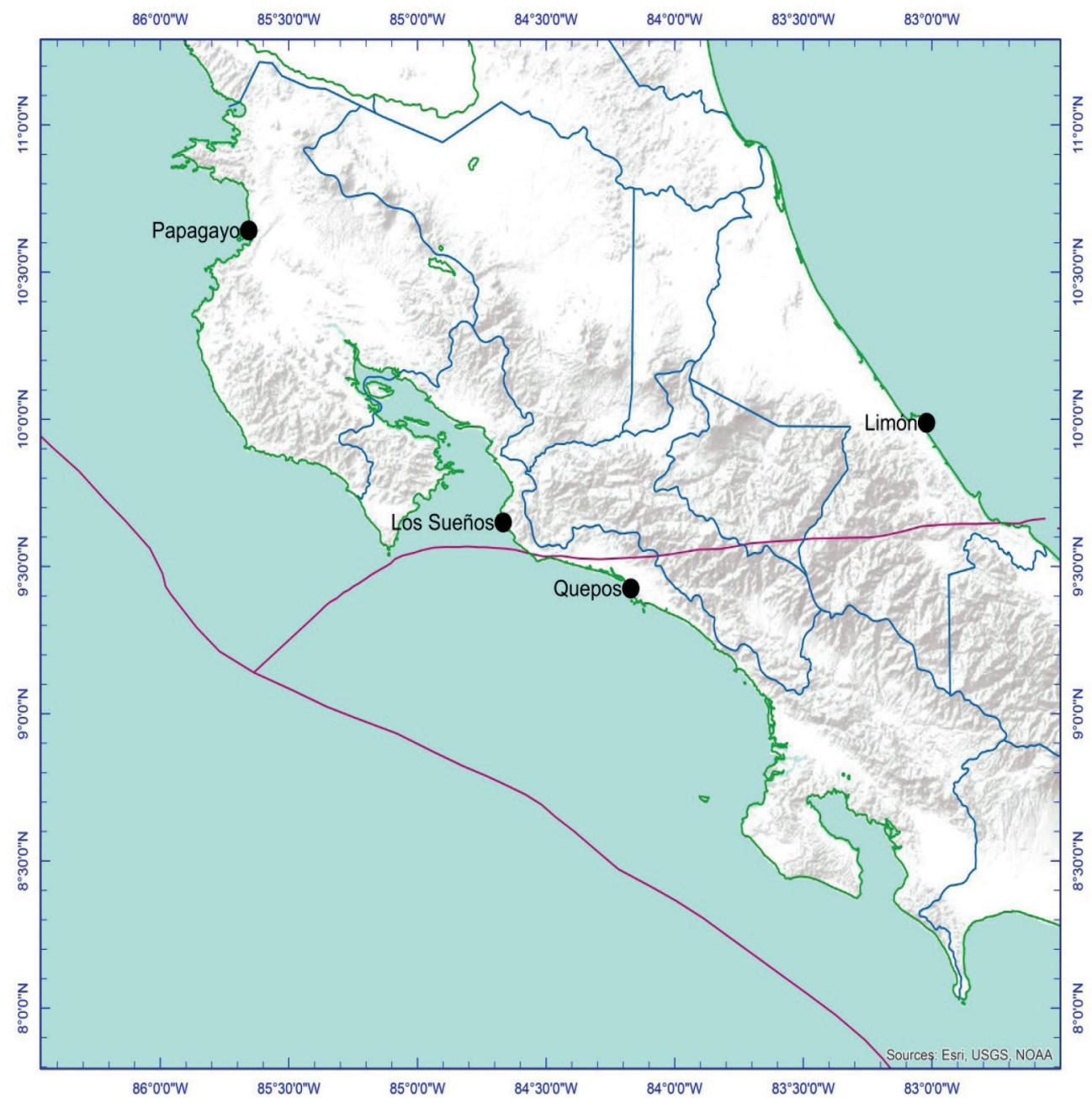

Fig. 8. Localización de las cuatro estaciones mareográficas que existen en Costa Rica, administradas por el Programa RONMAC. Las líneas rojas indican la localización aproximada de los bordes de las placas tectónicas de Cocos, Caribe y microplaca de Panamá Fig. 8. Location of the four tide gauge stations in Costa Rica, run by the RONMAC Program. Red lines indicate the approximate boundaries of the tectonic plates in Cocos, the Caribbean and the Panama microplate

La altura registrada del tsunami en Quepos corresponde con los rangos de alturas pronosticados tanto por el PTWC como por el SINAMOT.

El día 17 de setiembre en la mañana, el guardaparques Josué Morales de la Isla del Coco envió al SINAMOT un video del tsunami en la desembocadura del río Genio en la Isla, el cual se puede ver en la siguiente dirección: https://youtu.be/RePvCfoCTss Es posible observar como el tsunami "trepa" por el lecho del 


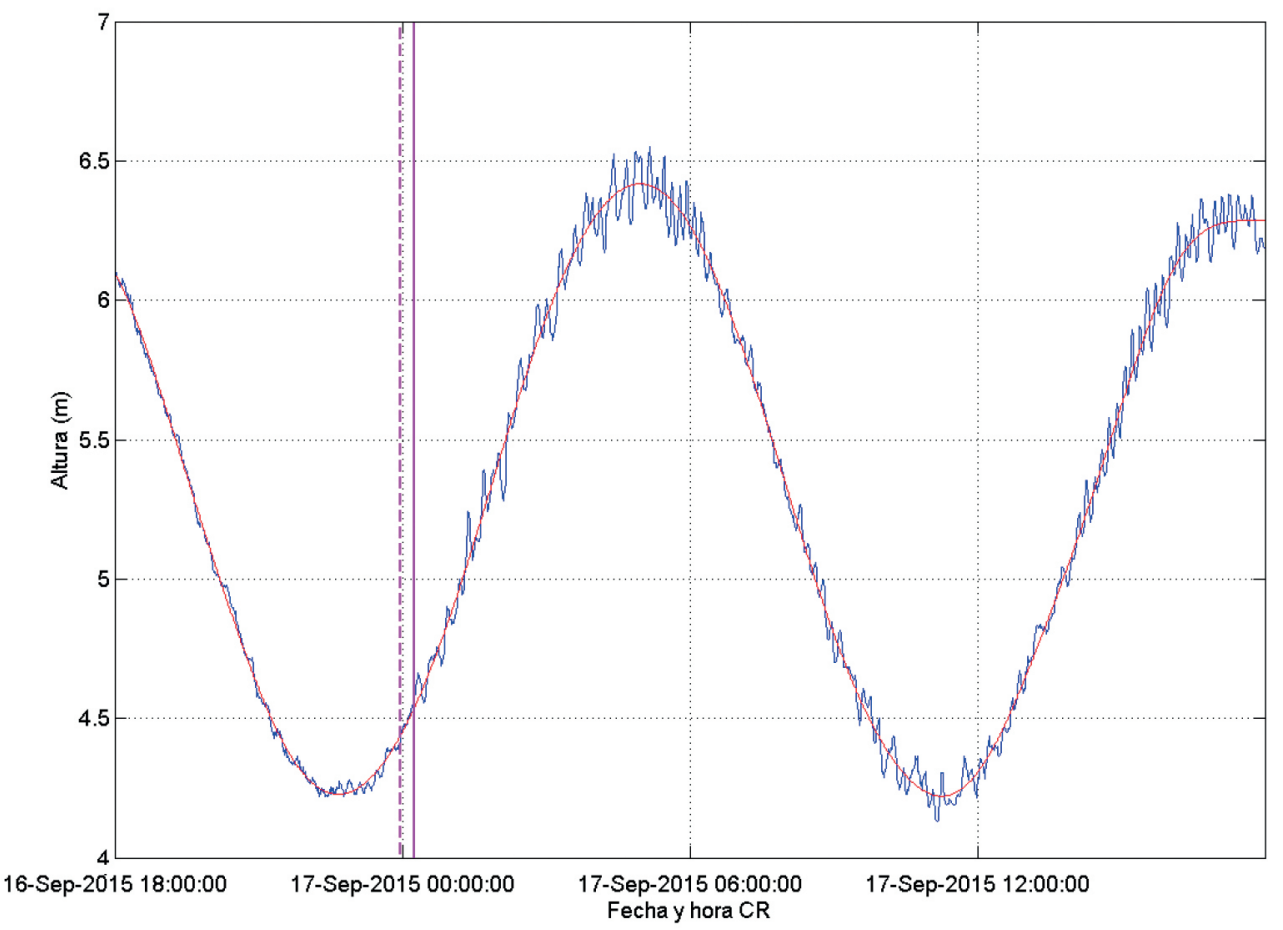

Fig. 9. Lectura del mareógrafo de Quepos de los días 16 y 17 de setiembre, hora local. La línea discontinua magenta indica la hora pronosticada de arribo. La línea continua magenta indica la hora en la que el tsunami arribó. La línea azul es el registro del mareógrafo y la línea roja indica la señal de la marea únicamente

Fig. 9. Record from the Quepos tide gauge station on September 16th and 17th, local time. Dashed magenta line shows the forecasted arrival time and the solid magenta line shows the tsunami arrival time for this station. The blue line is the tide gauge record and the red line shows solely the tide

río y si bien tiene alturas pequeñas, la velocidad de corriente que se puede ver es considerable. Este es el tercer registro de tsunami que tenemos en el mismo lugar, ya que se cuenta con un video similar del arribo del tsunami de Japón del 2011 y testimonios del arribo del tsunami de Chile del 2010. Con estos antecedentes resulta muy importante montar un modelo numérico de alta resolución para pronóstico de tsunamis en Bahía Wafer, Isla del Coco, que incluya parte del río Genio para futuros eventos, ya que por falta de embarcaderos los guardaparques utilizan dicho río para sacar y meter las embarcaciones al mar. Para esto ya se cuenta con algunos datos topográficos y batimétricos, cedidos por el Centro de Investigaciones en Ciencias del Mar y Limnología de la Universidad de Costa Rica (CIMAR-UCR), sin embargo, sería recomendable realizar un levantamiento batimétrico de mayor resolución en las cercanías de la desembocadura del río Genio. Adicionalmente, se manifiesta una vez más la necesidad de instalar una 


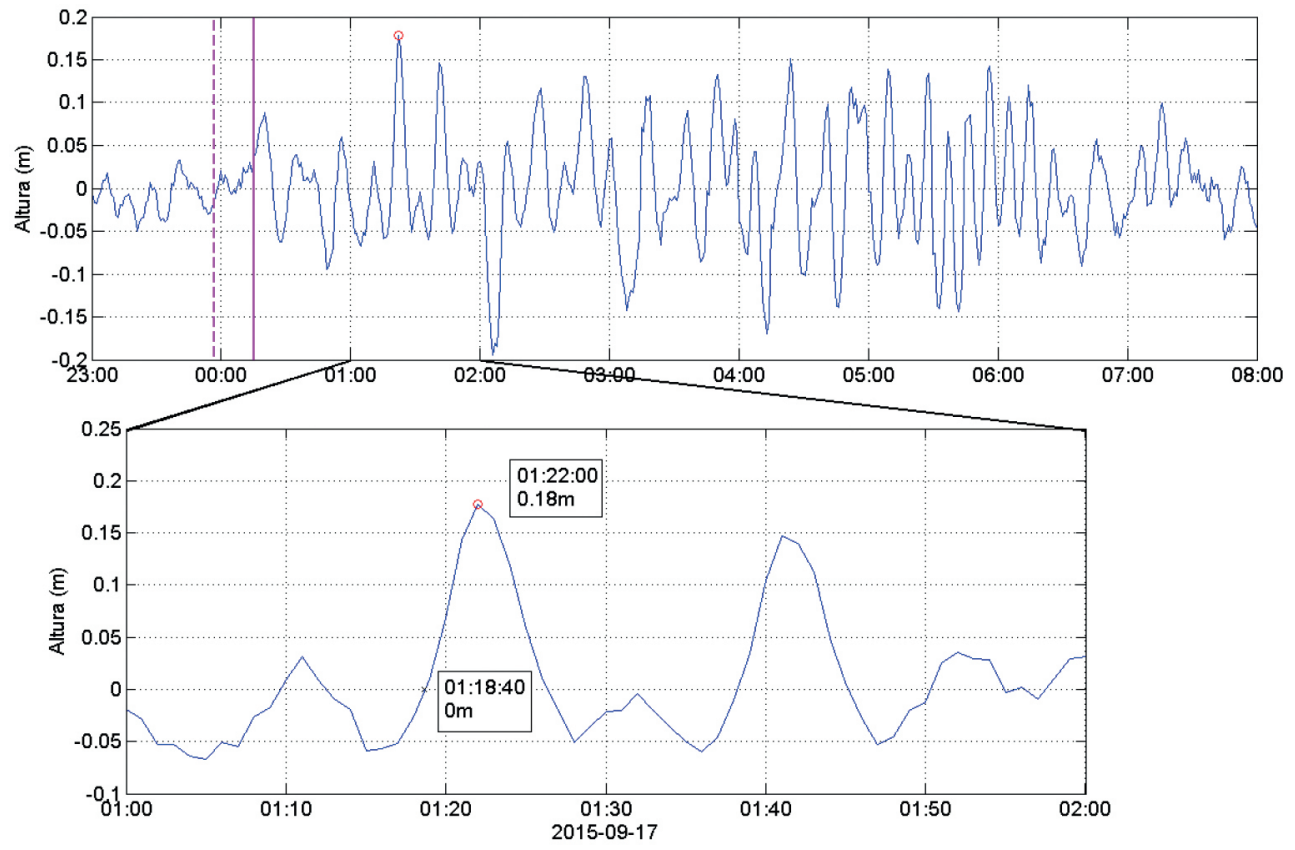

Fig. 10. Registro del tsunami en el mareógrafo de Quepos al que le fue eliminada la marea por medio de un filtro paso-alto (línea azul). La línea discontinua morada indica la hora pronosticada de arribo. La línea continua morada indica la hora en la que el tsunami arribó. El círculo rojo indica la altura máxima del tsunami que fue de $18 \mathrm{~cm}$ y se registró a la 1:22 a.m. del 17 de setiembre del 2015, hora local

Fig. 10. Detided tsunami record for the Quepos tide gauge station using a high-pass filter (blue line). Dashed purple line shows the forecasted arrival time. Solid purple line shows the tsunami arrival time for this station. The red circle shows the maximum tsunami height, which was $18 \mathrm{~cm}$ at 1:22 a.m. on September 17th, local time

estación del nivel del mar en la Isla, que se encuentra en negociaciones.

\section{CONCLUSIONES}

Este evento constituyó un ejercicio muy valioso que permitió poner a prueba los procedimientos y protocolos de análisis de alerta de tsunamis del SINAMOT con resultados exitosos.

Se calcularon horas del arribo aproximadas del tsunami a nuestro país. Se monitoreó el avance del tsunami a través del Pacífico, midiendo alturas máximas y períodos en las diferentes estaciones del nivel del mar que lo registraban. Se usaron registros históricos para saber que hasta el momento los tsunamis generados en Chile no han tenido consecuencias mayores para nuestro país. Se usaron las alturas costeras pronosticadas por el PTWC para confirmar el análisis y el pronóstico.

Se usaron los resultados del modelado publicados en el Tweb (NOAA) para ver la direccionalidad de la energía del tsunami, con lo que se pensó que podrían llegar corrientes peligrosas 
a la Isla del Coco. Se usó el modelo ComMIT para hacer un pronóstico de las alturas máximas y corrientes máximas del tsunami en nuestras costas, basado en la solución del Tweb con resultados exitosos. Dicho modelo estimó alturas máximas que fueron congruentes con las pronosticadas por el PTWC y con las registradas en el mareógrafo de Quepos. Este modelo constituye una herramienta muy importante, ya que en un tiempo razonable puede estimar cuáles regiones de la costa se verían más afectadas debido a un eventual tsunami grande, permitiendo así un adecuado enfoque de los recursos para la evacuación y atención de heridos y damnificados.

Sin embargo, es recomendable tener también modelos de pronóstico de alta precisión para al menos algunas localidades del país que se sabe presentan mayor peligrosidad. Una de ellas es la Isla del Coco, ya que debido a sus dimensiones y a la resolución de la malla en la que está incluida en el modelo de baja resolución se podría subestimar la afectación, sobre todo en las cercanías de la desembocadura del río Genio. No obstante, para establecer los modelos de pronóstico de alta precisión el SINAMOT necesitaría contar con más y mejor equipo de cómputo y en algunos casos mejores datos batimétricos, y más personal para que procese los resultados.

\section{AGRADECIMIENTOS}

Agradezco a la Universidad Nacional el apoyo para la realización de este estudio y a los revisores anónimos por las valiosas sugerencias. Así como a mis compañeros del SINAMOT por la asistencia brindada durante el evento.

\section{BIBLIOGRAFÍA}

British Oceanographic Data Center. (2011). General Bathymetry Chart of the Oceans. Recuperado el 15 octubre, 2015, disponible en: General Bathymetry Chart of the Oceans: http://www.gebco.net/

Chacón-Barrantes, S. \& Arozarena-Llopis, I. (in prep.). Tsunami threat for the Pacific Coast of Costa Rica: regional and far-field sources.

Chacón-Barrantes, S. \& Protti, M. (2011). Modeling a tsunami from the Nicoya, Costa Rica, seismic gap and its potential impact in Puntarenas. J. South Am. Earth Sci., 31, 372-382.

Farr, T. G., Rosen, P. A., Caro, E., Crippen, R., Duren, R., Hensley, S., . . \& \& Alsdorf, D. (2007). The Shuttle Rada Topography Mission. Rev. Geophysics, 45(2). doi:10.1029/2005RG000183

Geoware. (2012). Geoware Tsunami Product List. Recuperado el 15 octubre, 2015, disponible en: Geoware: http:// www.geoware-online.com/

INEGI. (2015). Tecnología LIDAR. Retrieved 15 october, 2015, from http:// www.inegi.org.mx/geo/contenidos/ geodesia/LiDAR.aspx

Levin, B. \& Nosov, M. (2009). Physics of Tsunamis. Moscow, Russia: Springer.

NGDC/WDS. (2015). Global Historical Tsunami Database. (National Geophysical Data Center / World Data Service) doi:10.7289/V5PN93H7. Recuperado el 23 setiembre, 2015, disponible en http:// www.ngdc.noaa.gov/hazard/tsu_db.shtml UNESCO. (2015). Pacific System (PTWS). Recuperado el 25 setiembre, 2015, disponible en: International Tsunami Information Center (ITIC): http://itic.iocunesco.org/index.php?option=com_co ntent $\&$ view $=$ category\&layout $=$ blog\&i $\mathrm{d}=1153 \&$ Itemid $=1153$ 